\title{
The Convergence of Galerkin Approximation Schemes for Second-Order Hyperbolic Equations With Dissipation
}

\author{
By Barbara Kok and Tunc Geveci
}

\begin{abstract}
In this paper we consider certain semidiscrete and fully discrete Galerkin approximations to the solution of an initial-boundary value problem for a second-order hyperbolic equation with a dissipative term. Estimates are obtained in the energy and negative norms associated with the problem, yielding in particular $H^{1}$ - and $L^{2}$-error estimates. The approximation to the initial data is taken, in this case, as the projection with respect to the energy inner product, onto the approximating space. We also obtain estimates for higher-order time derivatives.
\end{abstract}

1. Introduction. We consider the approximation of the solution of the initialboundary value problem

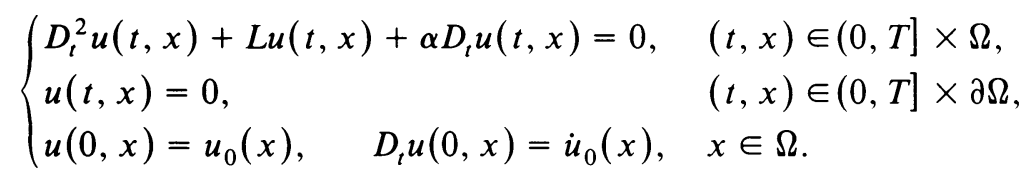

The domain $\Omega \subset \mathbf{R}^{N}$ is bounded with smooth boundary $\partial \Omega$. $L$ is the second-order elliptic operator

$$
L u=-\sum_{i, j=1}^{N} \frac{\partial}{\partial x_{i}}\left(a_{i j}(x) \frac{\partial u}{\partial x_{j}}\right)+a_{0}(x) u
$$

where

$$
a_{i j}=a_{j i} \in C^{\infty}(\bar{\Omega}) \quad \text { and } \quad \sum_{i, j} a_{i j}(x) \zeta_{i} \zeta_{j} \geqslant \beta|\zeta|^{2} \quad \forall x \in \bar{\Omega}, \forall \zeta \in \mathbf{R}^{N}, \beta>0 .
$$

We associate the bilinear form

$$
a(u, v)=\int_{\Omega}\left(\sum a_{i j} \frac{\partial u}{\partial x_{i}} \frac{\partial v}{\partial x_{j}}+a_{0} u v\right) d x
$$

with $L$. The term $\alpha D_{t} u(t, x), \alpha>0$, represents a retarding or frictional force which causes dissipation of energy.

Our aim is to extend the convergence analysis for conservative hyperbolic equations by Geveci [5] and Baker and Bramble [1] to include a dissipative term of the above form. 
The problem (1.1) and its approximation is considered in the framework of certain subspaces $\dot{H}^{s}(\Omega)$ of the Sobolev spaces $H^{s}(\Omega)$, as in [1], [2], [5] and [7]:

$$
\begin{aligned}
\dot{H}^{s}(\Omega)=\left\{v \in H^{s}(\Omega): L^{j} v\right. & =0 \text { on } \partial \Omega \text { for } j<s / 2\}, \quad s>0 . \\
\dot{H}^{\infty}(\Omega) & =\bigcap_{s \geqslant 0} \dot{H}^{s}(\Omega) .
\end{aligned}
$$

The eigenvalues of the operator $L$ with homogeneous Dirichlet boundary conditions form a sequence $\left\{\lambda_{j}\right\}_{j=1}^{\infty}$ of real, positive, nondecreasing numbers with corresponding orthonormal eigenfunctions $\left\{\varphi_{j}\right\}_{j=1}^{\infty}$, complete in $L^{2}(\Omega) . \dot{H}^{s}(\Omega)$ can then be defined equivalently as [4]

$$
\dot{H}^{s}(\Omega)=\left\{v \in L^{2}(\Omega):\|v\|_{s}=\left\{\sum_{j=1}^{\infty}\left|\left(v, \varphi_{j}\right)\right|^{2} \lambda_{j}^{s}\right\}^{1 / 2}<\infty\right\} .
$$

For $s>0, \dot{H}^{-s}(\Omega)$ denotes the dual of $\dot{H}^{s}(\Omega)$ with respect to the $L^{2}$-inner product, with norm

$$
\|v\|_{-s}=\left\{\sum_{j=1}^{\infty}\left|\left(v, \varphi_{j}\right)\right|^{2} \lambda_{j}^{-s}\right\}^{1 / 2}
$$

The solution operator $T: \dot{H}^{-1}(\Omega) \rightarrow \dot{H}^{1}(\Omega)$ of the associated elliptic boundary value problem is defined by

$$
a(T f, \varphi)=(f, \varphi) \quad \forall \varphi \in \dot{H}^{1}(\Omega),
$$

and is selfadjoint and positive definite on $L^{2}(\Omega)$ so that

$$
(v, w)_{-s} \equiv\left(T^{s} v, w\right)
$$

defines an inner product on $L^{2}(\Omega)$ (Thomée [7]).

In the formulation and presentation of the convergence analysis, we shall mainly follow Geveci [5]. Let $X$ denote the space $X \equiv \dot{H}^{1}(\Omega) \times L^{2}(\Omega)$ with the 'energy' inner product

$$
((U, V))_{0}=a(u, v)+(\dot{u}, \dot{v}) .
$$

For $U \in \dot{H}^{q+1}(\Omega) \times \dot{H}^{q}(\Omega)$, we have the norm $\|U\|_{q}^{2}=\|u\|_{q+1}^{2}+\|\dot{u}\|_{q}^{2}$. The initialboundary value problem can now be written as an evolution equation for $U(t)=$ $[u(t), \dot{u}(t)]^{T} \in X:$

$$
D_{t} U(t)+\Lambda_{\alpha} U(t)=0, \quad U(0)=U_{0}=\left[u_{0}, \dot{u}_{0}\right]^{T},
$$

where

$$
\Lambda_{\alpha}=\left[\begin{array}{cc}
0 & -I \\
L & \alpha I
\end{array}\right]=\Lambda+\alpha\left[\begin{array}{ll}
0 & 0 \\
0 & I
\end{array}\right]=\Lambda+\alpha \mathscr{I}
$$

where

$$
\Lambda=\left[\begin{array}{cc}
0 & -I \\
L & 0
\end{array}\right]
$$


as in [5], (1.5). We note that

$$
\Lambda^{q}=\left\{\begin{array}{cc}
(-1)^{q / 2}\left[\begin{array}{cc}
L^{q / 2} & 0 \\
0 & L^{q / 2}
\end{array}\right], & q \text { even } \\
(-1)^{(q-1) / 2}\left[\begin{array}{cc}
0 & -L^{(q-1) / 2} \\
L^{(q+1) / 2} & 0
\end{array}\right], & q \text { odd }
\end{array}\right.
$$

and

$$
\|U\|\left\|_{q}=\right\| \mid \Lambda^{q} U \|_{0} \quad([5,(1.8)]) .
$$

Dissipation of energy in the system (1.1) is demonstrated by the inequality

$$
\|U(t)\|_{0} \leqslant\|U(s) \mid\|_{0}, \quad t \geqslant s \geqslant 0,
$$

which is deduced from (1.2) as follows:

$$
\begin{aligned}
\frac{d}{d t}\|U(t) \mid\|_{0}^{2} & =\left(\left(D_{t} U(t), U(t)\right)\right)_{0}+\left(\left(U(t), D_{t} U(t)\right)\right)_{0} \\
& =\left(\left(-\Lambda_{\alpha} U(t), U(t)\right)\right)_{0}+\left(\left(U(t),-\Lambda_{\alpha} U(t)\right)\right)_{0} \\
& =a(\dot{u}, u)-a(u, \dot{u})-2 \alpha(\dot{u}, \dot{u})+a(u, \dot{u})-a(\dot{u}, u) \\
& =-2 \alpha(\dot{u}, \dot{u}) \leqslant 0 .
\end{aligned}
$$

Therefore,

$$
\|U(t)\|_{0}^{2} \leqslant\|U(s)\|_{0}^{2}, \quad t \geqslant s \geqslant 0 .
$$

Furthermore, for $U(t) \in \dot{H}^{q+1}(\Omega) \times \dot{H}^{q}(\Omega)$, we have the regularity result:

$$
\|U(t)\|_{q} \leqslant\|U(s) \mid\|_{q}, \quad t \geqslant s \geqslant 0 .
$$

In fact,

$$
\begin{aligned}
& \frac{d}{d t}\|U(t)\|_{q}^{2}=\frac{d}{d t}\left\|\Lambda^{q} U(t)\right\|_{0}^{2} \\
&=\left(\left(\Lambda^{q}(-\Lambda-\alpha \mathscr{I}) U, \Lambda^{q} U\right)\right)_{0}+\left(\left(\Lambda^{q} U, \Lambda^{q}(-\Lambda-\alpha \mathscr{I}) U\right)\right)_{0} \\
&=\left(\left(-\Lambda^{q+1} U, \Lambda^{q} U\right)\right)_{0}+\left(\left(\Lambda^{q} U,-\Lambda^{q+1} U\right)\right)_{0} \\
&-\alpha\left(\left(\Lambda^{q} \mathscr{I} U, \Lambda^{q} U\right)\right)_{0}-\alpha\left(\left(\Lambda^{q} U, \Lambda^{q} \mathscr{I} U\right)\right)_{0} \\
&=-\alpha\left(\left(\Lambda^{q}[0, \dot{u}], \Lambda^{q}[u, \dot{u}]\right)\right)_{0}-\alpha\left(\left(\Lambda^{q}[u, \dot{u}], \Lambda^{q}[0, \dot{u}]\right)\right)_{0} \\
&=-\alpha(-1)^{q}\left(\left(\Lambda^{2 q}[0, \dot{u}],[u, \dot{u}]\right)\right)_{0}-\alpha(-1)^{q}\left(\left(\Lambda^{2 q}[u, \dot{u}],[0, \dot{u}]\right)\right)_{0} \\
&=-\alpha\left(\left(\left[0, L^{q} \dot{u}\right],[u, \dot{u}]\right)\right)_{0}-\alpha\left(\left(\left[L^{q} u, L^{q} \dot{u}\right],[0, \dot{u}]\right)\right)_{0} \\
&=-2 \alpha\left(L^{q} \dot{u}, \dot{u}\right)=-2 \alpha\|\dot{u}\|_{q}^{2} \leqslant 0 .
\end{aligned}
$$

The semidiscrete Galerkin approximation to (1.1) is derived from the formulation

$$
\left(D_{t}^{2} u(t), \varphi\right)+a(u(t), \varphi)+\alpha\left(D_{t} u(t), \varphi\right)=0 \quad \forall \varphi \in \dot{H}^{1}(\Omega), u(t) \in \dot{H}^{1}(\Omega),
$$
i.e.,

$$
a\left(T D_{t}^{2} u(t), \varphi\right)+a(u(t), \varphi)+\alpha a\left(T D_{t} u(t), \varphi\right)=0 .
$$

Then, with $\dot{u}(t)=D_{t} u(t)$, we have

$$
T D_{t} \dot{u}(t)+u(t)+\alpha T \dot{u}(t)=0,
$$


and parallel to the treatment in [1] and [5], an evolution equation for $U(t)=$ $[u(t), \dot{u}(t)]^{T}$ :

$$
J D_{t} U(t)+U(t)+\alpha \mathscr{T} U(t)=0, \quad U(0)=U_{0}
$$

where

$$
J=\left[\begin{array}{cc}
0 & T \\
-I & 0
\end{array}\right], \quad \mathscr{T}=\left[\begin{array}{ll}
0 & T \\
0 & 0
\end{array}\right]
$$

We note that

$$
\begin{aligned}
& J: L^{2}(\Omega) \times \dot{H}^{-1}(\Omega) \rightarrow \dot{H}^{1}(\Omega) \times L^{2}(\Omega) \text { and } \\
& \mathscr{T}: \dot{H}^{1}(\Omega) \times L^{2}(\Omega) \rightarrow \dot{H}^{1}(\Omega) \times L^{2}(\Omega) .
\end{aligned}
$$

As in [5], we have

$$
\left\|\mid J^{p} U(t)\right\|\left\|_{0}=\right\|\|U(t)\|_{-p}, \quad p \geqslant 1,
$$

and we note that $J$ is skew-adjoint on $X$.

Semidiscrete Galerkin Scheme: Let $S_{h}^{r}(\Omega) \subset \dot{H}^{1}(\Omega)$ be a finite-dimensional subspace with the approximation property

$$
\inf _{\varphi_{h} \in S_{h}^{r}(\Omega)}\left\{\left\|u-\varphi_{h}\right\|_{0}+h\left\|u-\varphi_{h}\right\|_{1}\right\} \leqslant c h^{q}\|u\|_{q}, \quad 1 \leqslant q \leqslant r, r \geqslant 2,
$$

in which $h \in(0,1)$ is a parameter.

Let $T_{h}: \dot{H}^{-1}(\Omega) \rightarrow S_{h}^{r}(\Omega)$ be an operator approximating $T$ and be defined by

$$
a\left(T_{h} f, \varphi_{h}\right)=\left(f, \varphi_{h}\right) \quad \forall \varphi_{h} \in S_{h}^{r}(\Omega), f \in \dot{H}^{-1}(\Omega) .
$$

The operator $T_{h}$ has the properties (see [1] and [3]):

$$
T_{h} \text { is symmetric, positive semidefinite on } L^{2}(\Omega) \text { and positive }
$$$$
\text { definite on } S_{h}^{r}(\Omega) \text {; }
$$

and

$$
\left\|\left(T-T_{h}\right) f\right\|_{-p} \leqslant c h^{p+q+2}\|f\|_{q}, \quad-1 \leqslant p \leqslant r-2,-1 \leqslant q \leqslant r-2 .
$$

The semidiscrete approximation to the solution $u(t)$ of $(1.1)$ is a function $u_{h}(t) \in S_{h}^{r}(\Omega)$ which satisfies

$$
\begin{gathered}
\left(D_{t}^{2} u_{h}(t), \varphi_{h}\right)+a\left(u_{h}(t), \varphi_{h}\right)+\alpha\left(D_{t} u_{h}(t), \varphi_{h}\right)=0, \quad \varphi_{h} \in S_{h}^{r}(\Omega), t>0, \\
u_{h}(0)=u_{0, h} \in S_{h}^{r}(\Omega), \quad \dot{u}_{h}(0) \equiv D_{t} u_{h}(0)=\dot{u}_{0, h} \in S_{h}^{r}(\Omega) .
\end{gathered}
$$

We write this, in terms of $U_{h}(t)=\left[u_{h}(t), \dot{u}_{h}(t)\right]^{T}$, as

$$
\left\{\begin{array}{l}
J_{h} D_{t} U_{h}(t)+U_{h}(t)+\alpha \mathscr{T}_{h} U_{h}(t)=0, \quad t>0, \\
U_{h}(0)=U_{0, h},
\end{array}\right.
$$

where

$$
J_{h}=\left[\begin{array}{cc}
0 & T_{h} \\
-I & 0
\end{array}\right]: L^{2}(\Omega) \times H^{-1}(\Omega) \rightarrow S_{h}^{r}(\Omega) \times L^{2}(\Omega)
$$

is skew-adjoint in $S_{h}^{r}(\Omega) \times L^{2}(\Omega)$ (see [5]), and where

$$
\mathscr{T}_{h}=\left[\begin{array}{cc}
0 & T_{h} \\
0 & 0
\end{array}\right]: S_{h}^{r}(\Omega) \times L^{2}(\Omega) \rightarrow S_{h}^{r}(\Omega) \times L^{2}(\Omega) .
$$


If we define

$$
\|V\|_{-p, h}=\left\{\|v\|_{-(p-1), h}+\|v\|_{-p, h}\right\}^{1 / 2}, \quad p \geqslant 1,
$$

this being the seminorm induced by the bilinear form

$$
((V, W))_{-p, h} \equiv\left(T_{h}^{p-1} v, w\right)+\left(T_{h}^{p} \dot{v}, \dot{w}\right),
$$

we see that

(1.11) $\left\|\mid J_{h}^{p} U_{h}\right\|_{0}=\|\| U_{h} \|_{-p, h}, \quad p \geqslant 1$, for $U_{h} \in S_{h}^{r}(\Omega) \times L^{2}(\Omega) \quad[5]$.

Energy estimates are readily obtained from (1.10), parallel to the derivations of (1.4) and (1.5), in this case using the skew-adjointness of $J_{h}$ and

$$
J_{h}^{2 p}=(-1)^{p}\left[\begin{array}{cc}
T_{h}^{p} & 0 \\
0 & T_{h}^{p}
\end{array}\right]
$$

(see [5]). Thus we have

$$
\left\|\mid U_{h}(t)\right\|\left\|_{-p, h} \leqslant\right\| U_{h}(0)\|\|_{-p, h}, \quad p \geqslant 0, t>0 .
$$

We choose $U_{0, h}=\left[P_{h}^{1} u_{0}, P_{h}^{0} \dot{u}_{0}\right]^{T} \equiv \mathbf{P}_{h} U_{0}$, where $\mathbf{P}_{h}: \dot{H}^{1}(\Omega) \times L^{2}(\Omega) \rightarrow S_{h}^{r} \times S_{h}^{r}$ denotes the projection with respect to $((\cdot, \cdot))_{0}$, i.e.,

$$
\begin{aligned}
\left(P_{h}^{0} v, \varphi_{h}\right) & =\left(v, \varphi_{h}\right), & & \varphi_{h} \in S_{h}^{r}(\Omega), v \in L^{2}(\Omega), \text { and } \\
a\left(P_{h}^{1} v, \varphi_{h}\right) & =a\left(v, \varphi_{h}\right), & & \varphi_{h} \in S_{h}^{r}(\Omega), v \in \dot{H}^{1}(\Omega) .
\end{aligned}
$$

This is in accordance with the choice of initial data in [5].

Approximation-Theoretic Results: The following approximation-theoretic results are well known ([5] and [7]) and will be used repeatedly:

$$
\begin{aligned}
& \left\|v-P_{h}^{1} v\right\|_{-p} \leqslant c h^{p+q}\|v\|_{q}, \quad-1 \leqslant p \leqslant r-2,1 \leqslant q \leqslant r, \\
& \left\|v-P_{h}^{0} v\right\|_{-p} \leqslant c h^{p+q}\|v\|_{q} \quad 0 \leqslant p \leqslant r, 0 \leqslant q \leqslant r
\end{aligned}
$$

and hence,

$$
\left\|V-\mathbf{P}_{h} V\right\|_{-p} \leqslant c h^{p+q-1}\|V\|_{q-1}, \quad 0 \leqslant p \leqslant r-1,1 \leqslant q \leqslant r .
$$

We also have, from (1.9),

$$
\left\|\left(J-J_{h}\right) V \mid\right\|_{-p} \leqslant c h^{p+q-1}\|\| V \|_{q-2}, \quad 0 \leqslant p \leqslant r-1,1 \leqslant q \leqslant r,
$$

and

$$
\begin{aligned}
\|V\| \|_{-p, h} \leqslant c\left\{\|\| V\left\|_{-p}+h^{p}\right\| V \|_{0}\right\}, & V \in \dot{H}^{1}(\Omega) \times L^{2}(\Omega), \\
\|\| V \|_{-p} \leqslant c\left\{\|V\|_{-p, h}+h^{p}\|V\|_{0}\right\}, & 0 \leqslant p \leqslant r-1 .
\end{aligned}
$$

We now present the convergence analysis for the approximation of second-order hyperbolic equations with dissipation, following the ideas of Baker and Bramble [1], Geveci [5] and Thomée [7].

In Section 2 we obtain estimates in the energy and negative norms when the approximation to the initial data is the projection onto the approximating space $S_{h}^{r}(\Omega) \times S_{h}^{r}(\Omega)$, as defined by $(1.13)$.

In Section 3 we discuss fully discrete approximations generated by a class of 'acceptable' rational functions, as defined by Hersh and Kato [6].

In Section 4 we give estimates for the convergence of higher-order time derivatives in the semidiscrete case, that is, for $\left\|D_{t}^{s} U(t)-D_{t}^{s} U_{h}(t)\right\| \|_{-p}, 0 \leqslant p \leqslant r-1$. 
2. Convergence Estimates for Semidiscrete Approximations. The complete proofs of the results stated below appear in the supplements section at the end of this issue. The analysis therein follows that of Geveci [5, Section 2].

Proposition 1. If $U(t)$ satisfies (1.6) and $U_{h}(t)$ satisfies $(1.10)$ with $U_{h}(0)=\mathbf{P}_{h} U_{0}$, $U_{0} \in \dot{H}^{q+1}(\Omega) \times \dot{H}^{q}(\Omega)$, then

$$
\left\|U(t)-U_{h}(t)\right\|_{0} \leqslant c\left(t^{*}, \alpha\right) h^{q-1}\left\|U_{0}\right\|_{q}, \quad 1 \leqslant q \leqslant r, 0 \leqslant t \leqslant t^{*} .
$$

Proposition 2. If $U(t)$ satisfies (1.6) and $U_{h}(t)$ satisfies $(1.10)$ with $U_{h}(0)=\mathbf{P}_{h} U_{0}$ and $U_{0} \in \dot{H}^{q+1}(\Omega) \times \dot{H}^{q}(\Omega)$, then

$$
\left\|U(t)-U_{h}(t) \mid\right\|_{-p, h} \leqslant c\left(t^{*}, \alpha\right) h^{p+q-1}\left\|U_{0}\right\|_{q}, \quad 1 \leqslant p \leqslant r-1,1 \leqslant q \leqslant r .
$$

Propositions 1 and 2 then yield our main result.

THEOREM 1. If $U(t)$ is the solution of (1.6) and $U_{h}(t)$ is the solution of (1.10) with $U_{h}(0)=\mathbf{P}_{h} U_{0}, U_{0} \in \dot{H}^{q+1}(\Omega) \times \dot{H}^{q}(\Omega), 1 \leqslant q \leqslant r$, then for $0 \leqslant t \leqslant t^{*}$,

$$
\left.\left\|U(t)-U_{h}(t)\right\|\right|_{-p} \leqslant c\left(t^{*}, \alpha\right) h^{p+q-1}\left\|U_{0}\right\|_{q}, \quad 0 \leqslant p \leqslant r-1 .
$$

3. Convergence Estimates for Certain Fully Discrete Schemes. Let $I_{h}$ denote the identity map on $S_{h}^{r}(\Omega)$. The operator $T_{h}: \dot{H}^{-1}(\Omega) \rightarrow S_{h}^{r}(\Omega)$ is positive definite on $S_{h}^{r}(\Omega)$, so that we can define $L_{h}=\left(\left.T_{h}\right|_{S_{h}^{r}(\Omega)}\right)^{-1}$. Then as in [5, Section 3], we have

$$
\Lambda_{h} \equiv\left[\begin{array}{cc}
0 & -I_{h} \\
L_{h} & 0
\end{array}\right]=\left(\left.J_{h}\right|_{S_{h}^{r}(\Omega) \times S_{h}^{r}(\Omega)}\right)^{-1} .
$$

We can rewrite $(1.10)$ as

$$
D_{t} U_{h}(t)+\Lambda_{h} U_{h}(t)+\alpha \mathscr{I}_{h} U_{h}(t)=0, \quad U_{h}(0)=\mathbf{P}_{h} U_{0},
$$

where

$$
\mathscr{I}_{h}=\left[\begin{array}{cc}
0 & 0 \\
0 & I_{h}
\end{array}\right]
$$

Therefore,

$$
U_{h}(t)=e^{-\left(\Lambda_{h}+\alpha \mathscr{I}_{h}\right) t} \mathbf{P}_{h} U_{0} \equiv e^{-\Lambda_{\alpha, h} t} \mathbf{P}_{h} U_{0}
$$

We now construct single-step fully discrete approximations to (1.2). Let $r$ be a rational function such that [6]:

$$
\begin{gathered}
r(z)=e^{z}+O\left(|z|^{\nu+1}\right), \quad z \rightarrow 0, \quad \text { and } \\
|r(z)| \leqslant 1 \quad \text { for all } z \text { with } \operatorname{Re} z \leqslant 0 .
\end{gathered}
$$

The fully discrete approximation $\left\{W^{n}\right\}_{n=1}^{\infty} \subset S_{h}^{r}(\Omega) \times S_{h}^{r}(\Omega)$ to $U(t)$ is given by

$$
W^{n+1}=r\left[-k \Lambda_{\alpha, h}\right] W^{n}, \quad W^{0}=\mathbf{P}_{h} U_{0} ;
$$

i.e.,

$$
W^{n}=r^{n}\left[-k \Lambda_{\alpha, h}\right] \mathbf{P}_{h} U_{0},
$$

where $k$ is the time step. 
The following lemmas will be applied in the derivation of the error estimate for $U(t)-W^{n}, t=n k:$

We first note that $-\Lambda_{\alpha, h}$ is a dissipative operator, since for all $U \in S_{h}^{r}(\Omega) \times S_{h}^{r}(\Omega)$ we have

$$
\begin{aligned}
& \left(\left(U,-\Lambda_{\alpha, h} U\right)\right)_{0}=\left(\left([u, \dot{u}],\left[\dot{u},-L_{h} u-\alpha \dot{u}\right]\right)\right)_{0} \\
& \quad=-\alpha(\dot{u}, \dot{u})+a(u, \dot{u})-\overline{\left(L_{h} u, \dot{u}\right)}=-\alpha(\dot{u}, \dot{u})+a(u, \dot{u})-\overline{a\left(T_{h} L_{h} u, \dot{u}\right)} .
\end{aligned}
$$

Since $L_{h}=\left(\left.T_{h}\right|_{S_{h}^{r}}\right)^{-1}$ this reduced to $-\alpha(\dot{u}, \dot{u})+2 i \operatorname{Im} a(u, \dot{u})$. Therefore $\operatorname{Re}\left(\left(U,-\Lambda_{\alpha, h} U\right)\right)_{0} \leqslant 0$. The operator $-\Lambda_{\alpha, h}$ therefore generates a strongly continuous contraction semigroup.

LEMMA 1. Let $r$ be a rational function satisfying (3.3). Then

$$
\left\|r^{n}\left(-k \Lambda_{\alpha, h}\right)\right\|_{L\left(\mathbf{S}_{h}^{r}, \mathbf{S}_{h}^{r}\right)} \leqslant 1, \quad \text { where } \mathbf{S}_{h}^{r}=S_{h}^{r}(\Omega) \times S_{h}^{r}(\Omega) .
$$

Proof. This follows directly from Hersh and Kato [6, Theorem 6].

LEMMA 2. For all $f \in D\left(-\Lambda_{\alpha, h}^{\nu+1}\right)$ we have

$$
\left\|r^{n}\left(-k \Lambda_{\alpha, h}\right) f-e^{-n k \Lambda_{\alpha, h} f} \mid\right\|_{-p, h} \leqslant c\left(t^{*}\right) k^{\nu}\left\|\Lambda_{\alpha, h}^{\nu+1} f\right\|_{-p, h}, \quad p \geqslant 0 .
$$

Proof. See Hersh and Kato [6, Theorem 7].

LEMMA 3. For $2 \leqslant m \leqslant \nu+2, Z \in X$, we have

$$
\|\| \Lambda_{\alpha, h}^{m-1} J_{h}^{m} Z\left\|_{-p, h} \leqslant c(\alpha) \mid\right\| J_{h} Z \|_{-p, h}, \quad p \geqslant 0 .
$$

Proof. By definition

$$
\Lambda_{\alpha, h}^{m-1}=\left(\Lambda_{h}+\alpha \mathscr{I}_{h}\right)^{m-1} .
$$

We note that $\Lambda_{h} \mathscr{I}_{h}+\mathscr{I}_{h} \Lambda_{h}=\Lambda_{h} ; \Lambda_{h}^{2 j \mathscr{I}_{h}}=\mathscr{I}_{h} \Lambda_{h}^{2 j} \forall j \geqslant 1$ and $\mathscr{I}_{h}^{m}=\mathscr{I}_{h} \forall m \geqslant 1$. Then

$$
\left(\Lambda_{h}+\alpha \mathscr{I}_{h}\right)^{m-1}=\sum_{j=0}^{m-2} c_{m, j} \alpha^{j} \Lambda_{h}^{m-1-j}+\alpha^{m-1} \mathscr{I}_{h}+\sum_{j=1}^{\mu} d_{m, j} \alpha^{m-1-2 j \mathscr{I}_{h}} \Lambda_{h}^{2 j}
$$

where

$$
\begin{aligned}
& \mu= \begin{cases}(m-3) / 2 & \text { for } m \text { odd } \\
(m-2) / 2 & \text { for } m \text { even }\end{cases} \\
& c_{m, 0}=c_{m, m-3}=c_{m, m-2}=1 \text {; } \\
& \text { while for } 0<j<m-3, c_{m, j} \text { is determined by the triangle } \\
& m=2 \quad 1 \\
& m=3 \quad 11 \\
& 111 \\
& 1211 \\
& 12311 \\
& 133411 \\
& \text { and } d_{m, j}=c_{m, m-2-2 j}, \quad j=1, \ldots, \mu .
\end{aligned}
$$

On $S_{h}^{r}(\Omega) \times S_{h}^{r}(\Omega)$, we have

$$
\Lambda_{\alpha, h}^{m-1} J_{h}^{m-1}=\sum_{j=0}^{m-2} c_{m, j} \alpha^{j} J_{h}^{j}+\alpha^{m-1} \mathscr{I}_{h} J_{h}^{m-1}+\sum_{j=1}^{\mu} d_{m, j} \alpha^{m-1-2 j} \mathscr{I}_{h} J_{h}^{m-2 j-1}
$$


and for $Z \in X$,

$$
\begin{aligned}
\|\| \Lambda_{\alpha, h}^{m-1} J_{h}^{m-1}\left(J_{h} Z\right)\|\|_{-p, h} \leqslant & \sum_{j=0}^{m-2} c_{m, j} \alpha^{j}\left\|\left|J_{h}^{j}\left(J_{h} Z\right)\|\|_{-p, h}+\alpha^{m-1}\right|\right\| \mathscr{I}_{h} J_{h}^{m-1}\left(J_{h} Z\right) \|\left.\right|_{-p, h} \\
& +\sum_{j=1}^{\mu} d_{m, j} \alpha^{m-1-2 j}\left\|\mid \mathscr{I}_{h} J_{h}^{m-1-2 j}\left(J_{h} Z\right)\right\| \|_{-p, h} .
\end{aligned}
$$

For any $j \geqslant 0$

$$
\left\|\left|J_{h}^{j}\left(J_{h} Z\right)\left\|\left.\right|_{-p, h}=\right\|\left\|J_{h}^{p}\left(J_{h} Z\right)\right\|\left\|_{-j, h} \leqslant c\right\|\right|\left|J_{h}^{p}\left(J_{h} Z\right)\|\|_{0}=c\right|\right\| J_{h} Z\|\|_{-p, h} ;
$$

while

$$
\sum_{j=0}^{m-2} c_{m, j} \alpha^{j} \leqslant c(\alpha)
$$

Noting that $\left\|\left|\mathscr{I}_{h} W\|\|_{-p, h} \leqslant\left\|W|\||_{-p, h}, p \geqslant 0\right.\right.\right.$, we have, as above,

$$
\alpha^{m-1} \mid\left\|\mathscr{I}_{h} J_{h}^{m-1}\left(J_{h} Z\right)\right\|\left\|_{-p, h} \leqslant c(\alpha)\right\| J_{h}^{p}\left(J_{h} Z\right)\|\|_{0}=c(\alpha)\left\|J_{h} Z\right\| \|_{-p, h} ;
$$

and, similarly,

$$
\sum_{j=1}^{\mu} d_{m, j} \alpha^{m-1-2 j}\left|\left\|\mathscr{I}_{h} J_{h}^{m-2 j} Z||_{-p, h} \leqslant c(\alpha)\right\| J_{h} Z\right| \|_{-p, h} .
$$

The result follows.

As in [1] and [5], we define an auxiliary function $U_{0}^{(k)}=\left[u_{0}^{(k)}, \dot{u}_{0}^{(k)}\right]^{T}$, with $U_{0}^{(k)} \in \dot{H}^{\infty}(\Omega) \times \dot{H}^{\infty}(\Omega)$ and

$$
\begin{gathered}
\left\|U_{0}^{(k)}\right\|\left\|_{q+m} \leqslant k^{-m}\left|\left\|U_{0} \mid\right\|_{q},\right.\right. \\
\left\|U_{0}-U_{0}^{(k)}\right\|\left\|_{-p} \leqslant k^{q+p}\left|\left\|U_{0} \mid\right\|_{q}, \quad m, p, q \geqslant 0 .\right.\right.
\end{gathered}
$$

TheOrem 2. Assume $U_{0} \in\left(\dot{H}^{q+1}(\Omega) \times \dot{H}^{q}(\Omega)\right) \cap\left(\dot{H}^{s+1}(\Omega) \times \dot{H}^{s}(\Omega)\right)$. Then, for $2 \leqslant q \leqslant r, 2 \leqslant s \leqslant \nu+1, n k=t \leqslant t^{*}$

$$
\left\|W^{n}-U(n k)\right\| \|_{0} \leqslant c\left(t^{*}, \alpha\right)\left\{h^{q-1}\left|\left\|U_{0}\right\|_{q}+k^{s-1}\left\|\mid U_{0}\right\|_{s}\right\} .\right.
$$

\section{Proof. We first note that}

$$
\|\| W^{n}-U(n k)\left\|_{0} \leqslant\right\| W^{n}-U_{h}(n k)\|\|_{0}+\left\|U_{h}(n k)-U(n k)\right\|_{0} .
$$

From Proposition 1, we have

$$
\left\|U_{h}(n k)-U(n k)\right\|_{0} \leqslant c\left(t^{*}, \alpha\right) h^{q-1}\left\|U_{0}\right\|_{q},
$$

so that it remains for us to estimate

$$
\begin{aligned}
W^{n}-U_{h}(n k)= & r^{n}\left(-k \Lambda_{\alpha, h}\right) \mathbf{P}_{h} U_{0}-e^{-n k \Lambda_{\alpha, h}} \mathbf{P}_{h} U_{0} \\
= & \left\{r^{n}\left(-k \Lambda_{\alpha, h}\right)-e^{-n k \Lambda_{\alpha, h}}\right\} \mathbf{P}_{h} U_{0}^{(k)} \\
& +\left\{r^{n}\left(-k \Lambda_{\alpha, h}\right)-e^{-n k \Lambda_{\alpha, h}}\right\} \mathbf{P}_{h}\left(U_{0}-U_{0}^{(k)}\right) .
\end{aligned}
$$

We estimate the second term:

$$
\begin{aligned}
& \|\|\left\{r^{n}\left(-k \Lambda_{\alpha, h}\right)-e^{-n k \Lambda_{\alpha, h}}\right\} \mathbf{P}_{h}\left(U_{0}-U_{0}^{(k)}\right)\|\|_{0} \\
& \quad \leqslant\left\|r^{n}\left(-k \Lambda_{\alpha, h}\right)\right\|_{L\left(\mathbf{S}_{h}^{r}, \mathbf{S}_{h}^{r}\right)}\left\|\mathbf{P}_{h}\left(U_{0}-U_{0}^{(k)}\right)\right\|\left\|_{0}+\right\| \mathbf{P}_{h}\left(U_{0}-U_{0}^{(k)}\right)\|\|_{0} \\
& \quad \leqslant 2\left\|\mathbf{P}_{h}\left(U_{0}-U_{0}^{(k)}\right)\right\|\left\|_{0} \leqslant 2 k^{s-1}\right\| U_{0} \|_{s-1},
\end{aligned}
$$


by Lemma 1 and (3.6). Let $F_{n}(z)=r^{n}(z)-e^{n z}$. We now have to estimate

$$
\left\|F_{n}\left(-k \Lambda_{\alpha, h}\right) \mathbf{P}_{h} U_{0}^{(k)}\right\|_{0} \text {. }
$$

As in [1] and [5], we write

$$
U_{0}^{(k)}=\sum_{l=0}^{s} J_{h}^{l}\left(J-J_{h}\right) \Lambda^{l+1} U_{0}^{(k)}+J_{h}^{s+1} \Lambda^{s+1} U_{0}^{(k)}
$$

so that

$$
\begin{aligned}
\mathbf{P}_{h} U_{0}^{(k)}= & \mathbf{P}_{h}\left(J-J_{h}\right) \Lambda U_{0}^{(k)}+\mathbf{P}_{h} J_{h}\left(J-J_{h}\right) \Lambda^{2} U_{0}^{(k)}+\sum_{l=2}^{s} J_{h}^{l}\left(J-J_{h}\right) \Lambda^{l+1} U_{0}^{(k)} \\
& +J_{h}^{s+1} \Lambda^{s+1} U_{0}^{(k)} .
\end{aligned}
$$

However, $\mathbf{P}_{h}\left(J-J_{h}\right) Z=0, Z \in X$, and thus

$$
\begin{aligned}
\left\|F_{n}\left(-k \Lambda_{\alpha, h}\right) \mathbf{P}_{h} U_{0}^{(k)}\right\|_{0} \leqslant & \left\|F_{n}\left(-k \Lambda_{\alpha, h}\right) \mathbf{P}_{h} J_{h}\left(J-J_{h}\right) \Lambda^{2} U_{0}^{(k)}\right\|_{0} \\
& +\left\|F_{n}\left(-k \Lambda_{\alpha, h}\right) J_{h}^{s+1} \Lambda^{s+1} U_{0}^{(k)}\right\|_{0} \\
& +\sum_{l=2}^{s}\left\|F_{n}\left(-k \Lambda_{\alpha, h}\right) J_{h}^{l}\left(J-J_{h}\right) \Lambda^{l+1} U_{0}^{(k)}\right\|_{0} .
\end{aligned}
$$

By Lemma 1, (1.18), (1.17) and (3.5) the first term can be estimated as follows:

$$
\begin{aligned}
& \left\|F_{n}\left(-k \Lambda_{\alpha, h}\right) \mathbf{P}_{h} J_{h}\left(J-J_{h}\right) \Lambda^{2} U_{0}^{(k)}\right\|_{0} \\
& \quad \leqslant 2\left|\left\|J_{h}\left(J-J_{h}\right) \Lambda^{2} U_{0}^{(k)}\right\|\left\|_{0} \leqslant c h^{q}\right\|\right| \Lambda^{2} U_{0}^{(k)} \|_{q-2} \\
& \quad=c h^{q}\left\|U_{0}^{(k)}\right\|_{q} \leqslant c h^{q}\left\|U_{0}\right\|_{q} .
\end{aligned}
$$

For $2 \leqslant l \leqslant s$, we have by Lemmas 2 and 3, (1.18), (1.17) and (3.5),

$$
\begin{aligned}
& \left\|F_{n}\left(-k \Lambda_{\alpha, h}\right) J_{h}^{l}\left(J-J_{h}\right) \Lambda^{l+1} U_{0}^{(k)}\right\|_{0} \\
& \quad \leqslant c\left(t^{*}\right) k^{l-2}\left\|\mid \Lambda_{\alpha, h}^{l-1} J_{h}^{l}\left(J-J_{h}\right) \Lambda^{l+1} U_{0}^{(k)}\right\|_{0} \\
& \quad \leqslant c\left(t^{*}, \alpha\right) k^{l-2}\left\|J_{h}\left(J-J_{h}\right) \Lambda^{l+1} U_{0}^{(k)}\right\|_{0} \\
& \quad \leqslant c\left(t^{*}, \alpha\right) k^{l-2} h^{q-1}\left\|U_{0}^{(k)}\right\| \|_{q+l-2} \\
& \quad \leqslant c\left(t^{*}, \alpha\right) k^{l-2} h^{q-1} k^{-(l-2)}\left\|U_{0}\right\|_{q}=c\left(t^{*}, \alpha\right) h^{q-1}\left\|U_{0}\right\|_{q} .
\end{aligned}
$$

Finally, by Lemma $2(2 \leqslant s \leqslant \nu+1)$ and Lemma 3, it follows that

$$
\begin{aligned}
\left\|F_{n}\left(-k \Lambda_{\alpha, h}\right) J_{h}^{s+1} \Lambda^{s+1} U_{0}^{(k)} \mid\right\|_{0} & \leqslant c\left(t^{*}\right) k^{s-1}\left\|\mid \Lambda_{\alpha, h}^{s} J_{h}^{s+1} \Lambda^{s+1} U_{0}^{(k)}\right\| \|_{0} \\
& \leqslant c\left(\alpha, t^{*}\right) k^{s-1}\left\|J_{h} \Lambda^{s+1} U_{0}^{(k)}\right\|_{0} .
\end{aligned}
$$

We now apply result (3.35) of [5], namely

$$
\left\|J_{h} \Lambda^{s+1} U_{0}^{(k)}\right\|_{0} \leqslant c\left(\left\|U_{0}\right\|_{s}+k^{-(s-1)} h^{q-1}\left\|U_{0}\right\|_{q}\right),
$$

to obtain

(3.11) $\left\|F_{n}\left(-k \Lambda_{\alpha, h}\right) J_{h}^{s+1} \Lambda^{s+1} U_{0}^{(k)}\right\|_{0} \leqslant c\left(\alpha, t^{*}\right)\left\{k^{s-1}\left\|U_{0}\right\|\left\|_{s}+h^{q-1} \mid\right\| U_{0} \|_{q}\right\}$.

Combining (3.7)-(3.11) yields the result of the theorem.

We also establish negative norm estimates.

ThEOREM 3. For $2 \leqslant q \leqslant r, 2 \leqslant s \leqslant \nu+1,1 \leqslant p \leqslant r-1, n k=t \leqslant t^{*}$, we have

(3.12) $\left\|W^{n}-U_{h}(n k)|\||_{-p, h} \leqslant c\left(t^{*}, \alpha\right)\left\{h^{p+q-1}\left|\left\|U_{0}\left|\left\|_{q}+k^{s-1}\left|\left\|U_{0} \mid\right\|_{s-1}\right\}\right.\right.\right.\right.\right.\right.$,

(3.13) $\quad\left\|W^{n}-U_{h}(n k)\right\|_{-p}$

$$
\leqslant c\left(t^{*}, \boldsymbol{\alpha}\right)\left\{h^{p+q-1}\left\|U_{0}\right\|_{q}+\left(k^{s-1}+k^{s-2} h^{p}\right)\left\|U_{0}\right\| \|_{s-1}\right\},
$$




$$
\left.\left\|W^{n}-U_{h}(n k)\right\|\right|_{-p} \leqslant c\left(t^{*}, \alpha\right)\left\{h^{p+q-1}\left\|U_{0}\right\|_{q}+k^{s-1}\left\|U_{0}\right\| \|_{s}\right\} .
$$

Proof. The estimates (3.13) and (3.14) follow from (3.12), by using the energy estimate of Theorem 2 . In fact,

$$
\begin{aligned}
\left\|W^{n}-U_{h}(n k)\right\| \|_{-p} \leqslant & c\left\{\left.\left\|W^{n}-U_{h}(n k)\right\|\right|_{-p, h}+h^{p}\left\|W^{n}-U_{h}(n k)\right\| \|_{0}\right\} \\
\leqslant & c\left(t^{*}, \alpha\right)\left\{h^{p+q-1}\left\|U_{0}\right\|_{q}+k^{s-1}\left\|U_{0}\right\| \|_{s-1}\right\} \\
& +c\left(t^{*}, \alpha\right) h^{p}\left\{h^{q-1} \mid\left\|U_{0}\right\|_{q}+k^{s-2}\left\|U_{0}\right\| \|_{s-1}\right\} \\
\leqslant & c\left(t^{*}, \alpha\right)\left\{h^{p+q-1}\left\|U_{0}\right\|\left\|_{q}+\left(k^{s-1}+k^{s-2} h^{p}\right)\right\| U_{0} \|_{s-1}\right\} ;
\end{aligned}
$$

and

$$
\begin{aligned}
\left\|W^{n}-U_{h}(n k)\right\|_{-p} & \leqslant c\left(t^{*}, \alpha\right)\left\{h^{p+q-1}\left\|U_{0}\right\|_{q}+\left(k^{s-1}+k^{s-1} h^{p}\right)\left\|U_{0}\right\| \|_{s}\right\} \\
& \leqslant c\left(t^{*}, \alpha\right)\left\{h^{p+q-1}\left\|U_{0}\right\|_{q}+k^{s-1}\left\|U_{0}\right\|_{s}\right\} .
\end{aligned}
$$

We proceed to prove (3.12). As in the proof of Theorem 2, we write

$$
W^{n}-U_{h}(n k)=F_{n}\left(-k \Lambda_{\alpha, h}\right) \mathbf{P}_{h} U_{0}^{(k)}+F_{n}\left(-k \Lambda_{\alpha, h}\right) \mathbf{P}_{h}\left(U_{0}-U_{0}^{(k)}\right)
$$

and note that

(3.16) $\left\|\left|F_{n}\left(-k \Lambda_{\alpha, h}\right) \mathbf{P}_{h}\left(U_{0}-U_{0}^{(k)}\right)\right|\right\|_{-p, h} \leqslant 2\left\|\mathbf{P}_{h}\left(U_{0}-U_{0}^{(k)}\right)\right\|\left\|_{0} \leqslant c k^{s-1}\right\| U_{0} \|_{s-1}$ by (3.6). Estimating the first term of (3.15) is done as in Theorem 2 by

$$
\begin{aligned}
\left\|F_{n}\left(-k \Lambda_{\alpha, h}\right) \mathbf{P}_{h} U_{0}^{(k)}\right\| \|_{-p, h} \leqslant & \left\|F_{n}\left(-k \Lambda_{\alpha, h}\right) \mathbf{P}_{h} J_{h}\left(J-J_{h}\right) \Lambda^{2} U_{0}^{(k)}\right\| \|_{-p, h} \\
& +\sum_{l=2}^{s}\left\|F_{n}\left(-k \Lambda_{\alpha, h}\right) J_{h}^{l}\left(J-J_{h}\right) \Lambda^{l+1} U^{(k)}\right\| \|_{-p, h} \\
& +\left\|F_{n}\left(-k \Lambda_{\alpha, h}\right) J_{h}^{s+1} \Lambda^{s+1} U_{0}^{(k)} \mid\right\|_{-p, h} .
\end{aligned}
$$

Now, by (1.17) and (3.5) and noting that $J_{h} \mathbf{P}_{h} J_{h}=J_{h}^{2}$,

$$
\begin{aligned}
& \quad\left\|F_{n}\left(-k \Lambda_{\alpha, h}\right) \mathbf{P}_{h} J_{h}\left(J-J_{h}\right) \Lambda U_{0}^{(k)}\right\| \|_{-p, h} \\
& \quad \leqslant 2\left\|\mathbf{P}_{h} J_{h}\left(J-J_{h}\right) \Lambda U_{0}^{(k)}\right\|_{-p, h}=2\left\|\mid J_{h}^{p-1}\left(J_{h} \mathbf{P}_{h} J_{h}\right)\left(J-J_{h}\right) \Lambda^{2} U_{0}^{(k)}\right\|_{0} \\
& \quad=2\left\|\left(J-J_{h}\right) \Lambda^{2} U_{0}^{(k)}\right\|_{-(p+1), h} \leqslant c h^{p+q-1}\left\|U_{0}\right\|_{q-1} .
\end{aligned}
$$

For $l=2, \ldots, s$, we have

$$
\begin{aligned}
\left\|F_{n}\left(-k \Lambda_{\alpha, h}\right) J_{h}^{l}\left(J-J_{h}\right) \Lambda^{l+1} U_{0}^{(k)}\right\| \|_{-p, h} \\
\quad \leqslant c\left(t^{*}\right) k^{l-2}\left\|\Lambda_{\alpha, h}^{l-1} J_{h}^{l}\left(J-J_{h}\right) \Lambda^{l+1} U_{0}^{(k)}\right\|_{-p, h} \\
\quad \leqslant c\left(t^{*}, \alpha\right) k^{l-2}\left\|J_{h}\left(J-J_{h}\right) \Lambda^{l+1} U_{0}^{(k)}\right\|_{-p, h} \\
\quad=c\left(t^{*}, \alpha\right) k^{l-2}\left\|\left(J-J_{h}\right) \Lambda^{l+1} U_{0}^{(k)}\right\| \|_{-(p+1), h} \\
\quad \leqslant c\left(t^{*}, \alpha\right) k^{l-2} h^{p+q-1}\left\|\Lambda^{l+1} U_{0}^{(k)}\right\| \|_{q-3} \\
\quad \leqslant c\left(t^{*}, \alpha\right) k^{l-2} h^{p+q-1} k^{-(l-2)}\left\|U_{0}\right\|_{q} .
\end{aligned}
$$


Finally,

$$
\begin{aligned}
& \left\|F_{n}\left(-k \Lambda_{\alpha, h}\right) J_{h}^{s+1} \Lambda^{s+1} U_{0}^{(k)}\right\|\left\|_{-p, h} \leqslant c\left(t^{*}\right) k^{s-1}\right\| \Lambda_{\alpha, h}^{s} J_{h}^{s+1} \Lambda^{s+1} U_{0}^{(k)}\|\|_{-p, h} \\
& \leqslant c\left(t^{*}, \alpha\right) k^{s-1}\left\|J_{h} \Lambda^{s+1} U_{0}^{(k)}\right\|\left\|_{-p, h}=c\left(t^{*}, \alpha\right) k^{s-1}\right\| J_{h}^{p+1} \Lambda^{s+1} U_{0}^{(k)} \|_{0} .
\end{aligned}
$$

This is precisely (3.44) of [5], and applying the result (3.47) of the analysis there, we obtain

$$
\left\|F_{n}\left(-k \Lambda_{\alpha, h}\right) J_{h}^{s+1} \Lambda^{s+1} U_{0}^{(k)} \mid\right\|_{-p, h} \leqslant c\left(t^{*}, \alpha\right)\left\{k^{s-1}\left\|U_{0}\right\|\left\|_{s-1}+h^{p+q-1}\right\|\left\|U_{0}\right\|_{q}\right\} .
$$

The theorem is established by (3.16)-(3.19).

4. Estimates for Higher-Order Time Derivatives of Semidiscrete Approximations. To obtain energy and negative norm estimates for $D_{t}^{s} U(t)-D_{t}^{s} U_{h}(t)$, the following formulations of our evolution equations are used: $U(t)$ satisfies $((1.2))$

$$
D_{t} U(t)+\Lambda_{\alpha} U(t)=0, \quad U(0)=U_{0},
$$

where $\Lambda_{\alpha}=\Lambda+\alpha \mathscr{I}$. We can rewrite (1.6) as follows:

$$
\left(J+\alpha \mathscr{T}^{*}\right) D_{t} U(t)+U(t)=0, \quad U(0)=U_{0},
$$

where

$$
\mathscr{T}^{*}=\left[\begin{array}{ll}
T & 0 \\
0 & 0
\end{array}\right]
$$

Let $J_{\alpha} \equiv J+\alpha \mathscr{T}^{*}$. We note that $J_{\alpha} \Lambda_{\alpha}=I$.

The semidiscrete approximation $U_{h}(t)$ satisfies $((3.1))$

$$
D_{t} U_{h}(t)+\Lambda_{\alpha, h} U_{h}(t)=0, \quad U_{h}(0)=U_{0, h},
$$

where $\Lambda_{\alpha, h}=\Lambda_{h}+\alpha \mathscr{I}_{h}$. One can also rewrite (1.10) as

$$
\left(J_{h}+\alpha \mathscr{T}_{h}^{*}\right) D_{t} U_{h}(t)+U_{h}(t)=0, \quad U_{h}(0)=U_{0, h}
$$

where

$$
\mathscr{T}_{h}^{*}=\left[\begin{array}{cc}
T_{h} & 0 \\
0 & 0
\end{array}\right]
$$

Let $J_{\alpha, h} \equiv J_{h}+\alpha \mathscr{T}_{h}^{*}$ and note that $J_{\alpha, h} \Lambda_{\alpha, h}=I$ on $S_{h}^{r}(\Omega) \times S_{h}^{r}(\Omega)$. The following result will be needed:

$$
\left\|\left|\Lambda_{\alpha}^{m} Z\right|\right\|_{p} \leqslant c(\alpha)\left|\|Z \mid\|_{m+p}, \quad m \geqslant 1, p \geqslant 0 .\right.
$$

In fact, as in Lemma 3,

$$
\Lambda_{\alpha}^{m}=\sum_{j=0}^{m-1} c_{m, j} \alpha^{j} \Lambda^{m-j}+\alpha^{m} \mathscr{I}+\sum_{j=1}^{\mu} d_{m, j} \alpha^{m-2 j \mathscr{I}} \Lambda^{2 j}
$$

where

$$
\mu= \begin{cases}(m-1) / 2 & \text { for } m \text { odd } \\ (m-2) / 2 & \text { for } m \text { even, }\end{cases}
$$

and the coefficients $c_{m, j}$ and $d_{m, j}$ are as defined in Lemma 3. For $Z \in \dot{H}^{p+m+1}(\Omega)$ $\times \dot{H}^{p+m}(\Omega)$, we have

$$
\Lambda^{p} \Lambda_{\alpha}^{m} Z=\sum_{j=1}^{m-1} c_{m, j} \alpha^{j} \Lambda^{m+p-j} Z+\alpha^{m} \Lambda^{p \mathscr{I}} Z+\sum_{j=1}^{\mu} d_{m, j} \alpha^{m-2 j} \Lambda^{p} \mathscr{I} \Lambda^{2 j} Z,
$$


so that

$$
\begin{aligned}
\left\|\Lambda_{\alpha}^{m} Z\right\|_{p} & \equiv\left\|\Lambda^{p} \Lambda_{\alpha}^{m} Z\right\|_{0} \\
& \leqslant \sum_{j=0}^{m-1} c_{m, j} \alpha^{j}\left\|\Lambda^{m+p-j} Z\right\|_{0}+\alpha^{m}\left\|\mid \Lambda^{p \mathscr{I}} Z\right\|_{0}+\sum_{j=1}^{\mu} d_{m, j} \alpha^{m-2 j}\left\|\Lambda^{p \mathscr{I}} \Lambda^{2 j} Z\right\|_{0} \\
& \leqslant\left(\sum_{j=0}^{m-1} c_{m, j} \alpha^{j}\right)\left\|\Lambda^{m+p} Z\right\|_{0}+\alpha^{m}\|\mathscr{I} Z\|_{p}+\sum_{j=1}^{\mu} d_{m, j} \alpha^{m-2 j}\left\|\mathscr{I} \Lambda^{2 j} Z\right\|_{p} \\
& \leqslant c(\alpha)\left\{\|Z\|_{m+p}+\|Z Z\|_{p}+\|Z\| \|_{p+\left\{\begin{array}{l}
m-1 \\
m-2
\end{array}\right\}}\right\} \leqslant c(\alpha)\|Z\|_{m+p} .
\end{aligned}
$$

Analogous to [2] and [5], we shall choose $U_{h}(0)=J_{\alpha, h}^{s+1} \Lambda_{\alpha}^{s+1} U_{0}, s \geqslant 1$. Our main result is

TheOREM 4. Assume $U_{0} \in \dot{H}^{s+q+1}(\Omega) \times \dot{H}^{s+q}(\Omega), s \geqslant 1,1 \leqslant q \leqslant r$. Then

$$
\left\|D_{t}^{s} U(t)-D_{t}^{s} U_{h}(t)\right\|_{-p} \leqslant c\left(t^{*}, \alpha\right) h^{p+q-1}\left\|U_{0}\right\|_{s+q}, \quad 0 \leqslant p \leqslant r-1 .
$$

The proof can be found in the supplements section at the end of this issue.

National Research Institute for Mathematical Sciences

Council for Scientific and Industrial Research

P. O. Box 395

Pretoria 0001, Republic of South Africa

1. G. A. BAKER \& J. H. Bramble, "Semidiscrete and single step fully discrete approximations for second order hyperbolic equations," RAIRO Anal. Numér., v. 13, 1979, pp. 75-100.

2. G. A. BAKER \& V. A. Dougalis, "On the $L^{\infty}$-convergence of Galerkin approximations for second-order hyperbolic equations," Math. Comp., v. 34, 1980, pp. 401-424.

3. J. H. Bramble, A. H. Schatz, V. Thomee \& L. B. Wahlbin, "Some convergence estimates for semidiscrete Galerkin type approximations for parabolic equations," SIAM J. Numer. Anal., v. 14, 1977, pp. $218-241$.

4. J. H. Bramble \& V. ThomeE, "Discrete time Galerkin methods for a parabolic boundary value problem," Ann. Mat. Pura. Appl. (4), v. 101, 1974, pp. 115-152.

5. T. GEVECI, "On the convergence of Galerkin approximation schemes for second-order hyperbolic equations in energy and negative norms," Math. Comp., v. 42, 1984, pp. 393-415.

6. R. HerSH \& T. KATO, "High-accuracy stable difference schemes for well-posed initial-value problems,” SIAM J. Numer. Anal., v. 16, 1979, pp. 670-682.

7. V. THOMEE, “Negative norm estimates and superconvergence in Galerkin methods for parabolic problems," Math. Comp., v. 34, 1980, pp. 93-113. 


\title{
Supplement to The Convergence of Galerkin Approximation Schemes for Second-Order Hyperbolic Equations With Dissipation
}

\author{
By Barbara Kok and Tunc Geveci
}

2. Convergence Estimates for Semi-discrete Approximations

PROPOSITION 1. If $U(t)$ satissies (1.6) and $U_{h}(t)$ satisfics $(1.10)$

with $U_{h}(0)=P_{h} U_{0}, \quad U_{0} c A^{q+1}(\Omega) \times A^{q}(\Omega)$ then

$$
\|\| U(t)-U_{h}(t)\left\|_{0} \leq c\left(t^{*}, \alpha\right) h^{q-1}\right\| U_{0} \mid \|_{q}, \quad \begin{array}{ll}
1 & \leq q \leq r \\
& 0 \leq t \leq t^{*} .
\end{array}
$$

Proos. Firstly for $q=1$, we have

$$
\begin{aligned}
\|\| U(t)-U_{h}(t) ! \|_{0} & \leq\|\| U(t)\|\|_{0}+\|\| U_{h}(t) \| !_{0} \\
& \leq\|\| U_{0}\|!\|_{0}+\|\| \mathbf{P}_{h} U_{0}\|\|_{0} \\
& \leq 2\|\| U_{0}\|\|_{0}
\end{aligned}
$$

by (1.4), (1.12) and since $\mathbf{P}_{\mathrm{h}}$ is the projection with respect to $((., .))_{0}$. For $2 \leq q \leq r$, we write

$$
U(t)-U_{h}(t)=\left(U(t)-P_{h} U(t)\right)+\left(\mathbf{P}_{h} U(t)-U_{h}(t)\right) .
$$

By (1.16),

$$
\begin{aligned}
\|\| U(t)-P_{h} U(t) \| i_{0} & \leq c h^{q-1}\|i U(t)\|_{q-1} \\
& \leq c h^{q-1}\left\|U_{0} \cdot\right\|_{q-1} \\
& \leq c h^{q-1}\|\| U_{0} \cdot \|_{q}
\end{aligned}
$$

we have to sho:l that

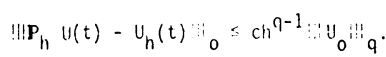



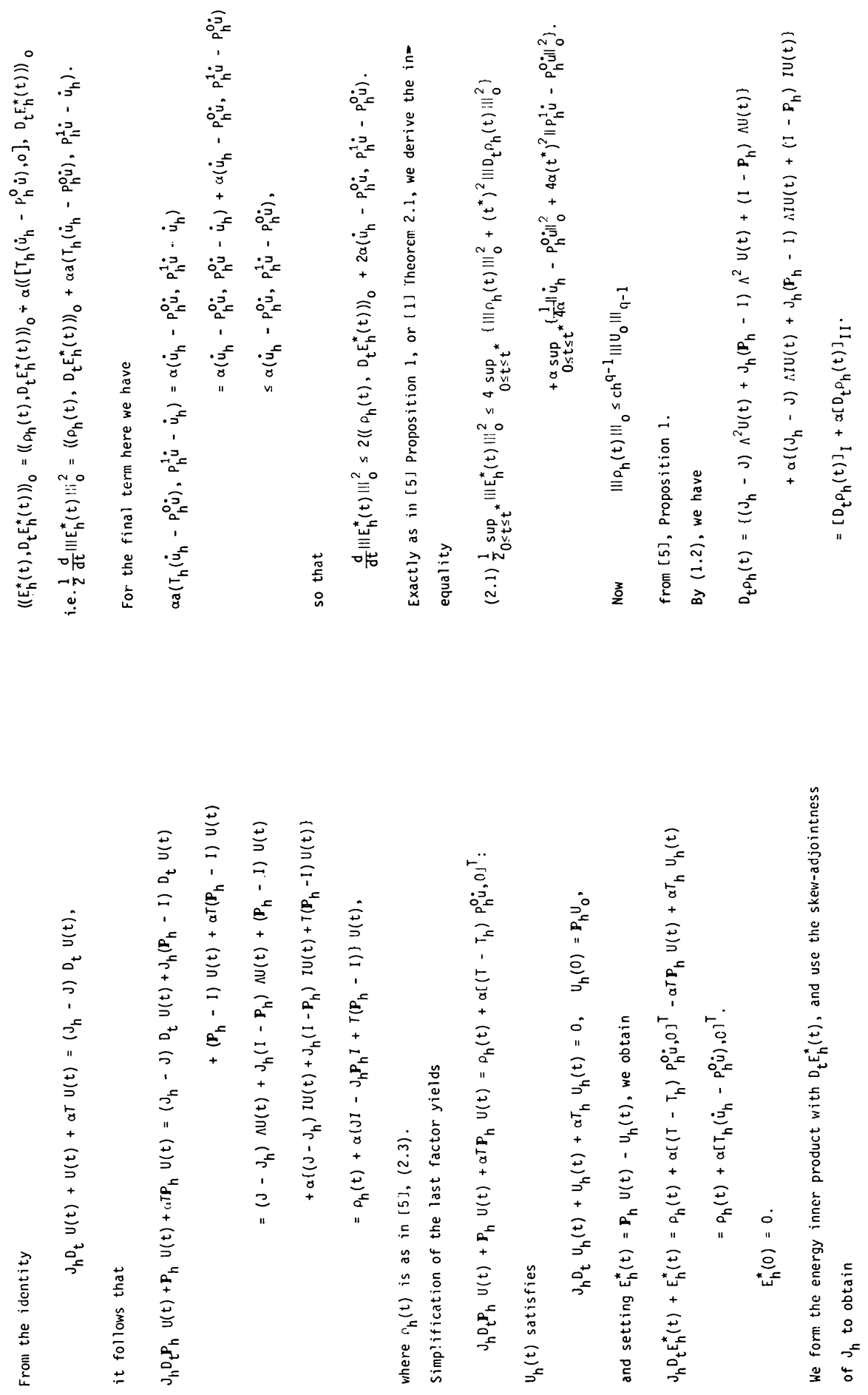

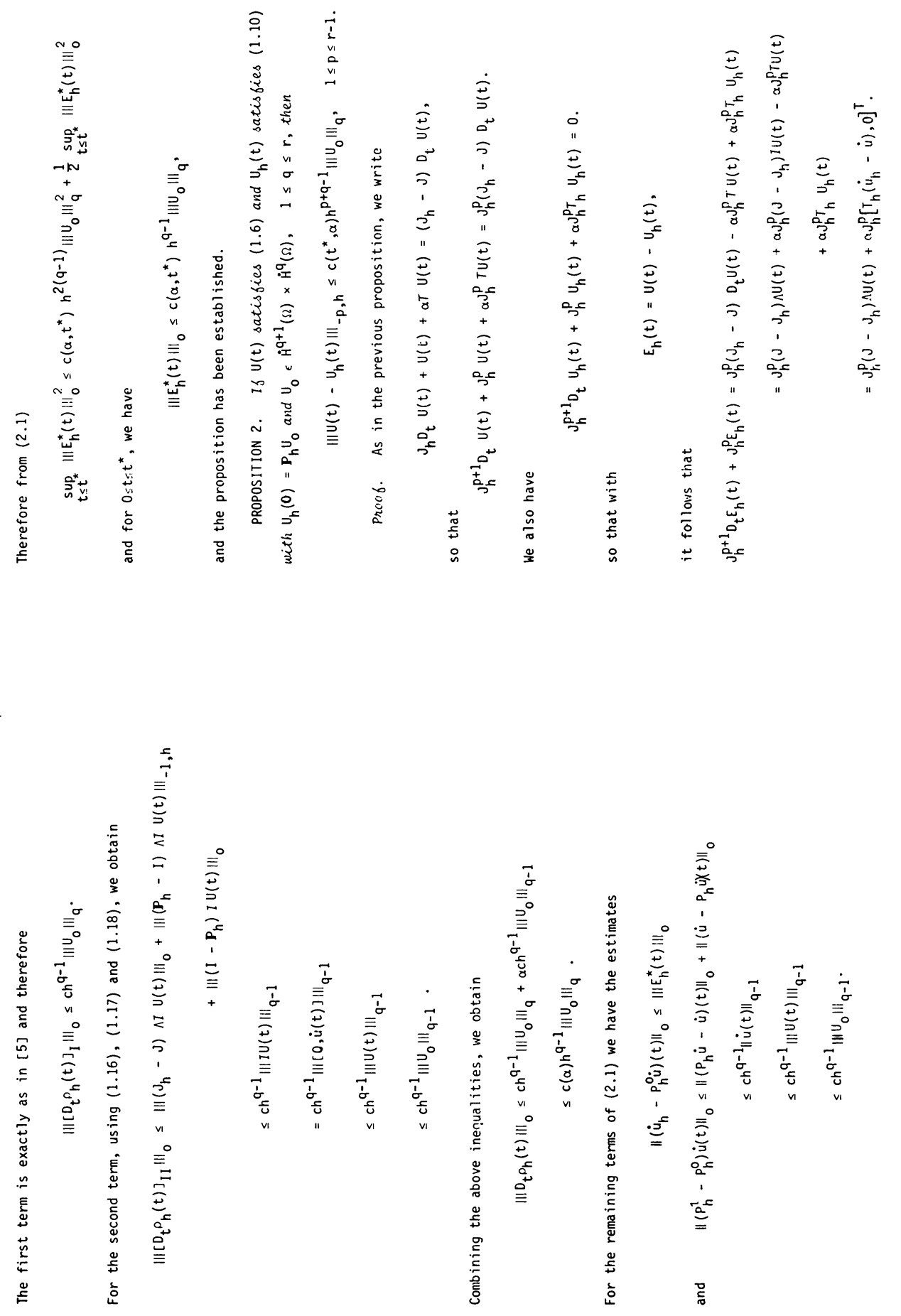

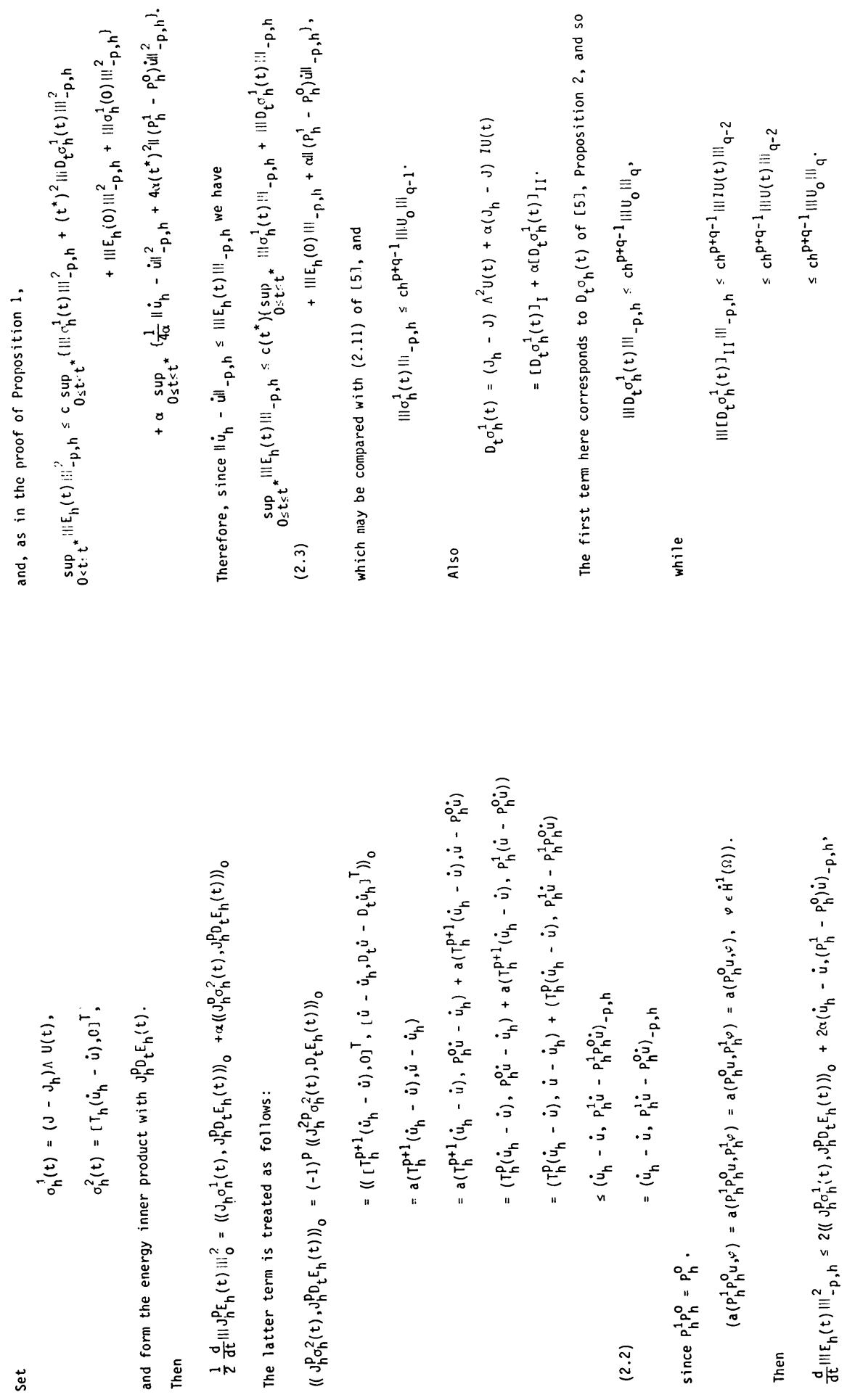

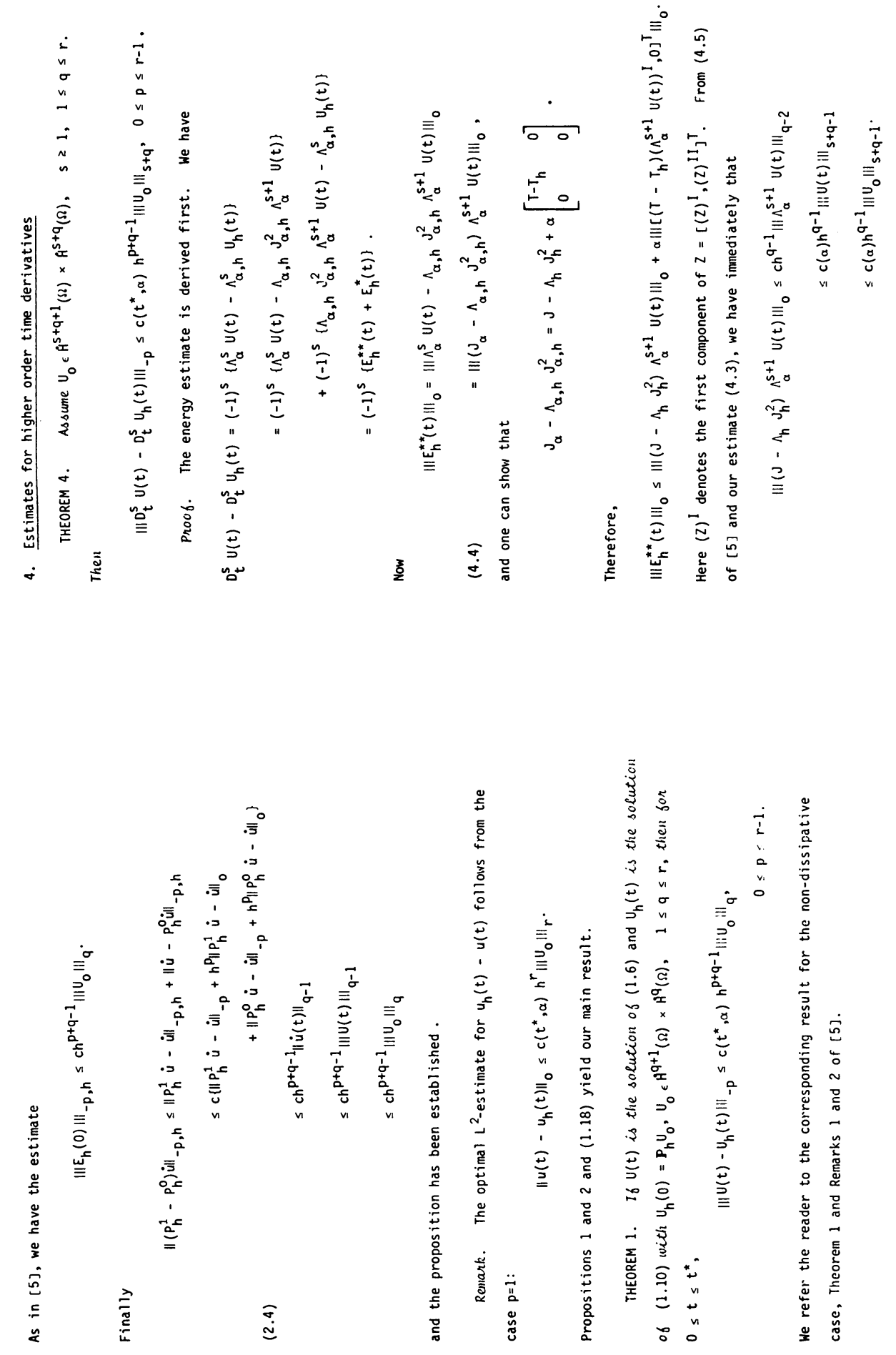

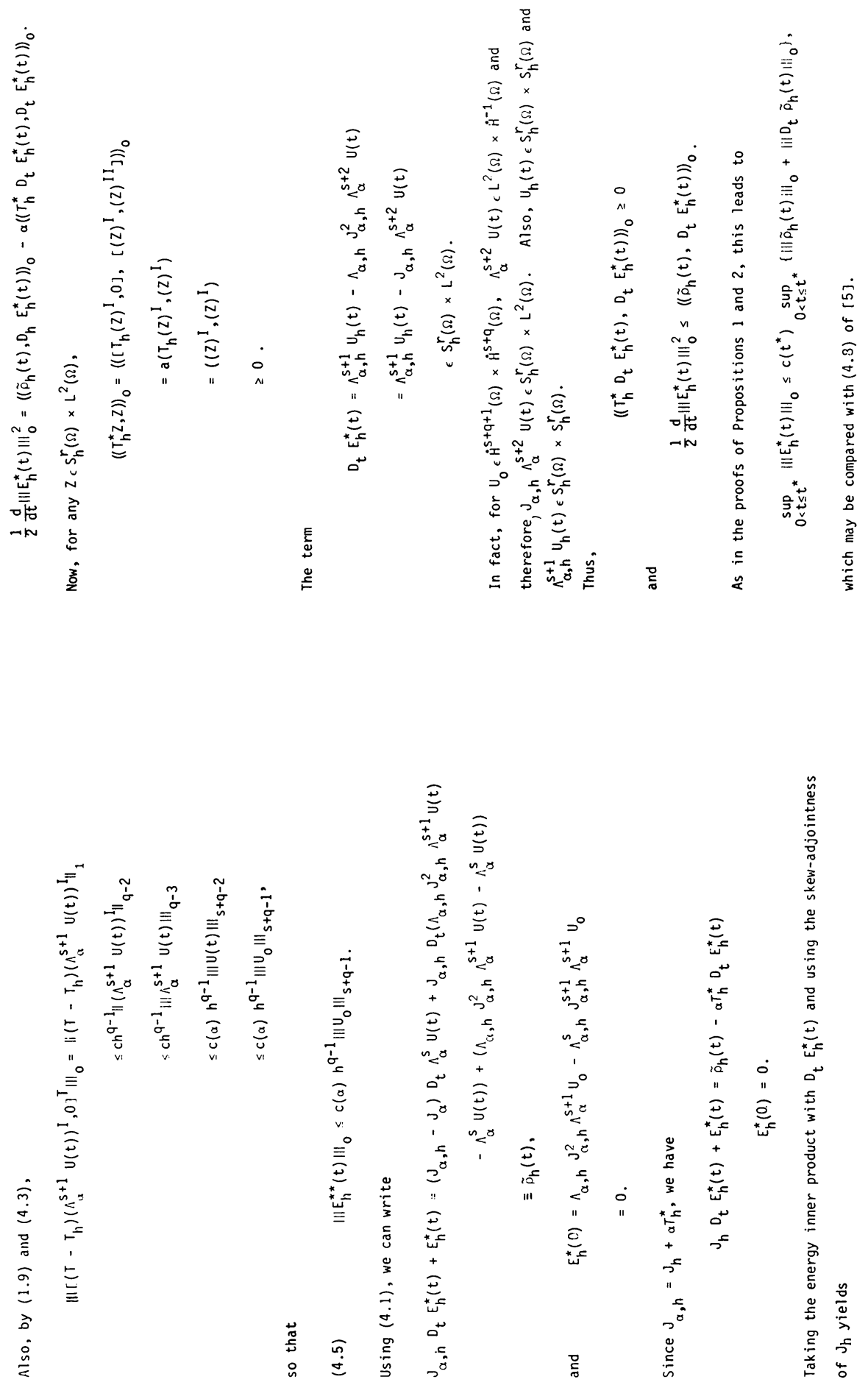

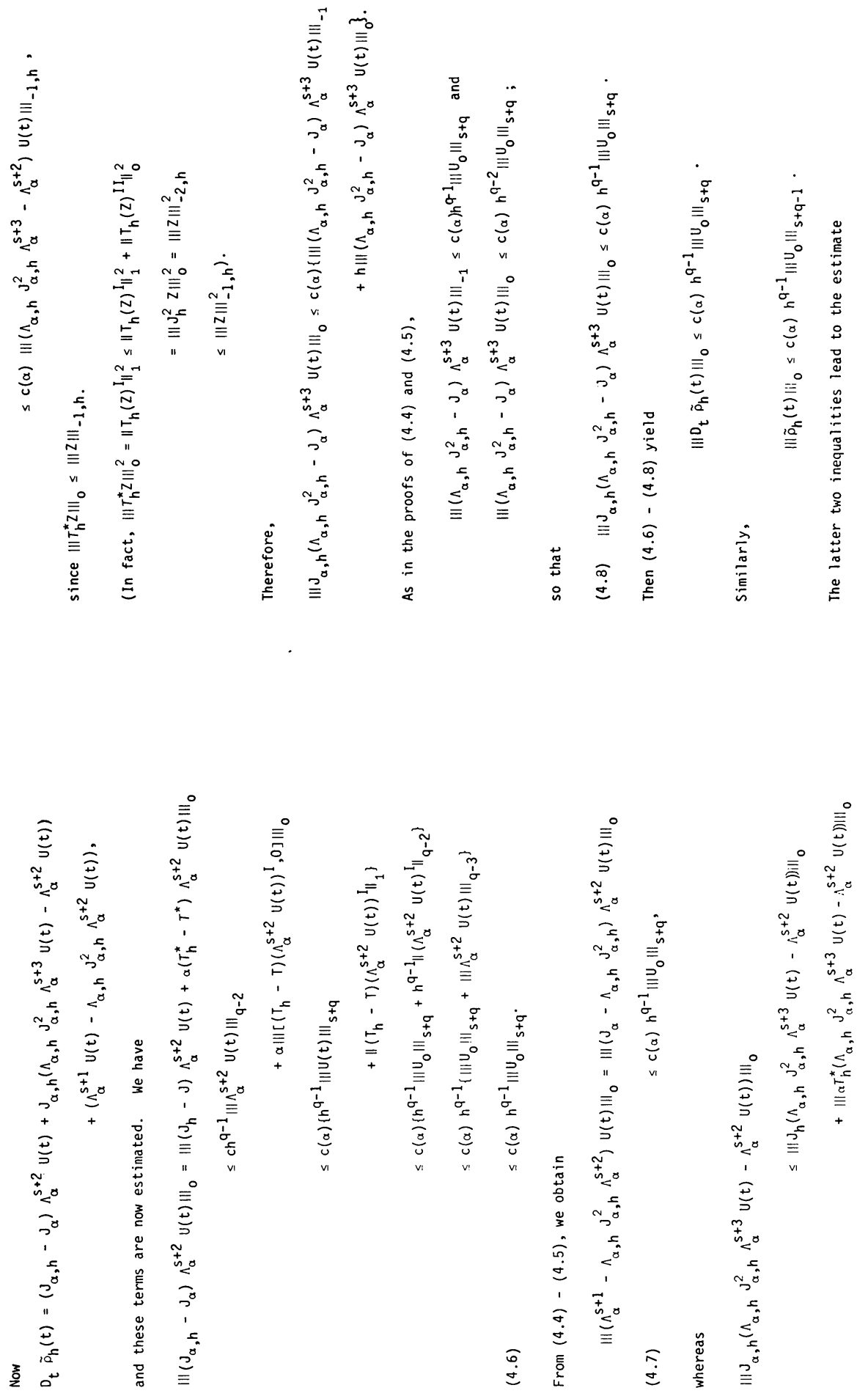

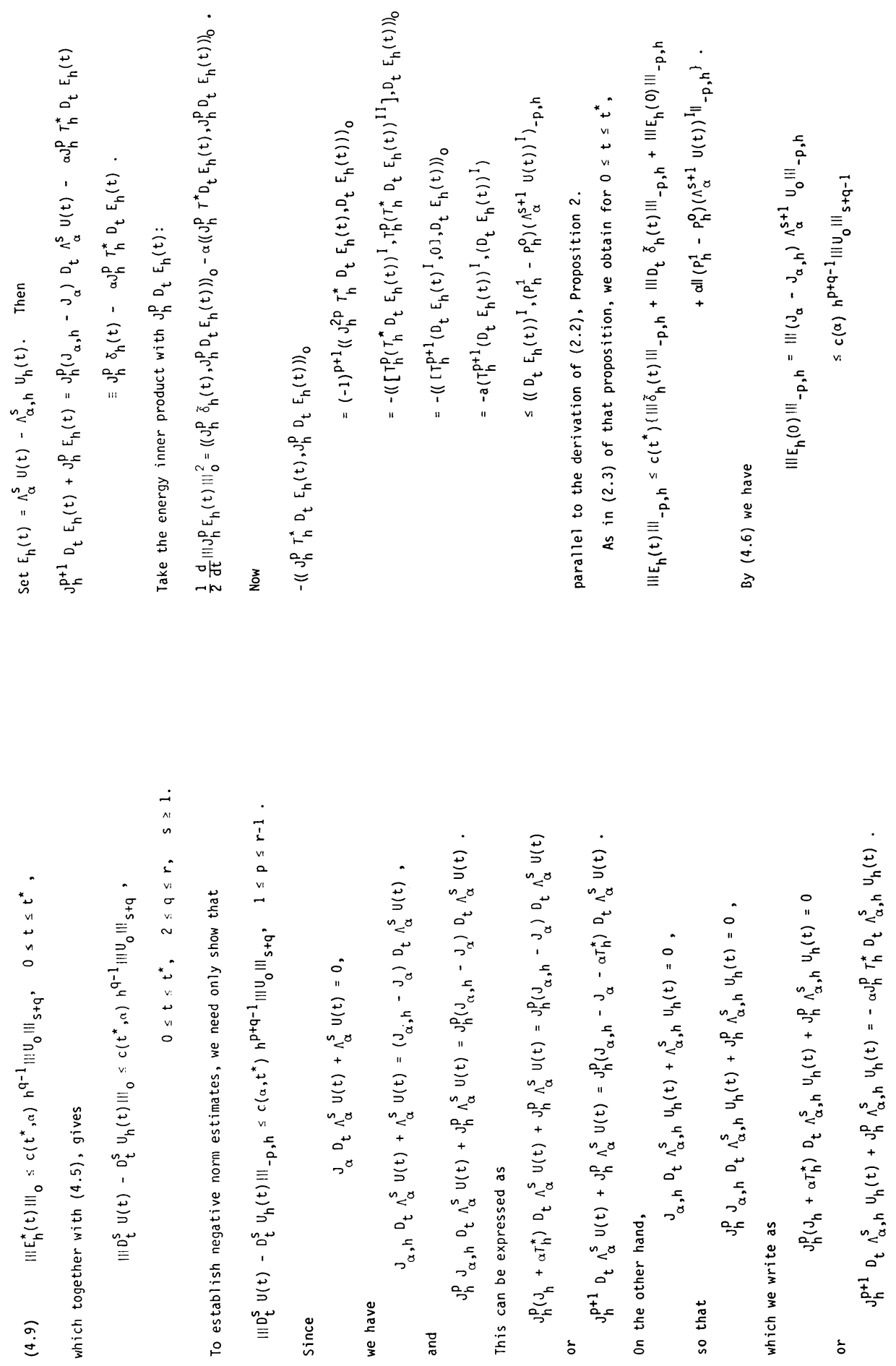


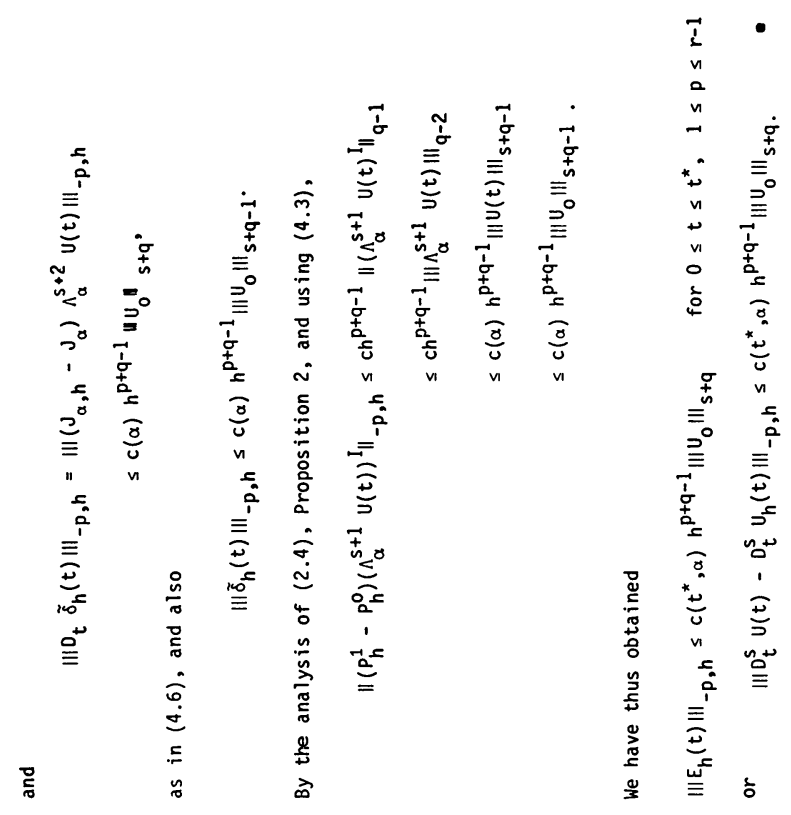

